\title{
A utilização do sistema CAD/CAM na resolução estética anterior de paciente com diferentes substratos
}

The use of the CAD/CAM system in the previous aesthetic resolution of a patient with different substrates

El uso del sistema CAD/CAM en la resolución estética previa de un paciente con diferentes sustratos

Leonardo Almeida

ORCID: https://orcid.org/0000-0002-2593-0664 Universidade Estadual do Oeste do Paraná, Brasil E-mail: leonardoalmeida.tem@gmail.com

Stefany Couto Santana

ORCID: https://orcid.org/0000-0001-7770-5197 Universidade Estadual do Oeste do Paraná, Brasil E-mail: stefanycouto@hotmail.com

Fabiana Scarparo Naufel ORCID: https://orcid.org/0000-0003-0486-8512 Universidade Estadual do Oeste do Paraná, Brasil E-mail: biberes@terra.com.br

Vera Lúcia Schmitt

ORCID: https://orcid.org/0000-0002-9539-4174 Universidade Estadual do Oeste do Paraná, Brasil E-mail: veraschmitt@uol.com.br

Lucinara Ignez Tavares Luzzi

ORCID: https://orcid.org/0000-0003-1397-6590 Universidade Estadual do Oeste do Paraná, Brasil E-mail: lucinaratluzzi@gmail.com

Adriano Piccolotto

ORCID: https://orcid.org/0000-0003-4230-5649 Universidade Estadual do Oeste do Paraná, Brasil E-mail: adriano_pccltt@hotmail.com

\begin{abstract}
Resumo
Atualmente na odontologia, existem tratamentos que aliam não só estética e longevidade, mas também facilidade na execução e consequentemente economia de tempo. O objetivo deste trabalho é demonstrar, através de um relato de caso clínico, a capacidade do sistema CAD/CAM CEREC (Ceramic Reconstruction) na mimetização da estrutura dental e padronização do substrato dental no resultado final. O presente estudo relata a confecção de restaurações anteriores (13, 12, 11, 21, 22 e 23) em dissilicato de lítio (E.max - Ivoclar vivadent), em uma paciente do gênero feminino, 50 anos, com diferentes substratos dentais, através de sistemas completos para consultório (chairside). Como resultados obtevese: etapas clínicas alcançadas com sucesso, ótimas adaptações das peças, mimetização das estruturas dentais, resultando em restaurações cerâmicas de excelência, bem indicadas, confiáveis e esteticamente agradáveis, que foi o principal motivo pelo qual a paciente procurou atendimento.
\end{abstract}

Palavras-chave: CAD-CAM; Cerâmicas; Cor; Estética dentária; Reabilitação bucal.

\begin{abstract}
Currently in dentistry, there are treatments that combine not only aesthetics and longevity, but also ease of execution and consequently saving time. The objective of this work is to demonstrate, through a clinical case report, the ability of the CAD/CAM CEREC (Ceramic Reconstruction) system to mimic the dental structure and standardize the dental substrate in the final result. The present study reports the preparation of previous restorations $(13,12,11,21,22$ and 23) in lithium disilicate (e.max - Ivoclar vivadent), in a female patient, 50 years old, with different dental substrates, through complete office systems (chair side). As results obtained: clinical steps successfully achieved, excellent adaptations of the pieces, mimicment of the dental structures, results in ceramic restorations of excellence, well indicated, qualified and aesthetically pleasing, which was the main reason why the patient attended.
\end{abstract}

Keywords: CAD-CAM; Ceramics; Color; Esthetics dental; Mouth rehabilitation. 


\section{Resumen}

Actualmente en odontología existen tratamientos que combinan no solo estética y longevidad, sino también facilidad de ejecución y consecuentemente ahorro de tiempo. El objetivo de este trabajo es demostrar, a través de un caso clínico, la capacidad del sistema CAD/CAM CEREC (Ceramic Reconstruction) para imitar la estructura dental y estandarizar el sustrato dental en el resultado final. El presente estudio reporta la preparación de restauraciones previas $(13,12,11$, 21, 22 y 23) en disilicato de litio (E.max - Ivoclar vivadent), en una paciente de 50 años, con diferentes sustratos dentales, a través de sistemas de oficina (lado de la silla). Como resultados obtenidos: pasos clínicos logrados con éxito, excelentes adaptaciones de las piezas, mimetismo de las estructuras dentales, resulta en restauraciones cerámicas de excelencia, bien indicadas, calificadas y estéticamente agradables, que fue la principal razón por la que el paciente asistió.

Palabras clave: CAD-CAM; Céramiques; Color; Dentisterie esthétique; Rehabilitación bucal.

\section{Introdução}

Ao longo dos anos, as próteses odontológicas se reformularam de maneira significativa, melhorando consigo a tão famosa tríade da odontologia. Com essa evolução, o perfil do paciente também se desenvolveu, e ele passou a se tornar muito mais exigente com o tratamento a ser recebido.

As cerâmicas odontológicas evoluíram em resistência e, consequentemente, na função, além da longevidade, estética e biocompatibilidade (Alves et al., 2017).

A tecnologia $\mathrm{CAD} / \mathrm{CAM}$ também teve um grande desenvolvimento quanto à leitura dos preparos, digitalização e na manufatura das restaurações, revolucionando assim a odontologia restauradora (Correia et al., 2006).

Os sistemas CAD/CAM, se referem basicamente ao desenho de uma peça protética guiado por um computador (Computer Aided Design), seguido da sua manufatura por fresagem (Computer Aided Manufaturing). Este, teve sua incorporação à odontologia no final da década de 70 .

A incorporação das tecnologias CAD/CAM tem algumas características que merecem e devem ser salientadas; é uma tecnologia que torna os profissionais já capacitados em profissionais mais rápidos e eficazes, o trabalho mais produtivo, preciso e, consequentemente, mais rentável (Moura et al., 2015).

Esses sistemas são compostos por três componentes, sendo um scanner intra-oral, que é usado para a digitalização de estruturas orais, modelos ou impressões (Alves et al., 2017). Um software que permite o planejamento e desenho da restauração e uma fresadora ou unidade CAM, que é responsável pela confecção da restauração ou infraestrutura. O maior ganho da utilização deste sistema é a precisão da adaptação nas restaurações indiretas (Correia et al., 2006).

Os sistemas cerâmicos em geral apresentam alta dureza e baixa resistência flexural, que pode variar de 20 a $60 \mathrm{MPa}$, chegando a $150 \mathrm{MPa}$. Já os sistemas a base de dissilicato de lítio apresentam boa resistência flexural, chegando a valores próximos a $360 \mathrm{MPa}$ (conforme fabricante), permitindo assim, restaurações com menor risco de fratura (Martins, 2012).

Um requisito essencial de uma restauração de cerâmica pura é sua habilidade de mascaramento, sem a qual irá permitir a exibição inaceitável de um dente escurecido ou um pino metálico abaixo de uma restauração translúcida (Correia et al., 2006) (Moura et al., 2015). Para mascarar a cor subjacente ou alterar a cor final de uma restauração, controlar a opacidade e a saturação torna-se muito importante. A cor e a opacidade são fatores-chave, e devem estar em harmonia para se obter resultados altamente estéticos (Moura et al., 2015). A habilidade de mascaramento das cerâmicas deve-se, em grande parte, às propriedades ópticas da microestrutura e da espessura relativa do sistema de infraestrutura/estratificação. Assim, o aumento de espessura da cerâmica, a utilização de cimentos resinosos com diferentes cores ou a indicação de sistemas cerâmicos adequados têm sido sugeridos como maneiras de mascarar o substrato desfavorável (Martins, 2012).

Um material cerâmico melhorado, fabricado a partir da IPS Empress 2, o IPS e.max Press ou CAD (Ivoclar Vivadent, Liechtenstein), foi introduzido em 2005 e consiste de palhetas ou blocos cerâmicos com diferentes cores. Há uma variedade de opacidades disponíveis (HO, MO, LT, MT e HT) para esse material, sendo possível sua utilização para confecção de infra- 
estruturas, inclusive na presença de substratos escurecidos, como alternativa à utilização de infraestruturas em zircônia (Martins, 2012).

Além disso, o dissilicato de lítio ainda apresenta boa biocompatibilidade, resistência à compressão, condutibilidade térmica semelhante aos tecidos dentais, estabilidade de cor e, principalmente, elevado potencial para mimetizar dentes, por possuir um índice de refração semelhante ao esmalte dental (Chang, et al., 2009).

\section{Metodologia}

O presente estudo refere-se a um relato de caso e análise de literaturas publicadas, em bases de dados eletrônicas nacionais e internacionais: BVS (Biblioteca Virtual de Saúde), SciELO (Scientific Electronic Library Online), PUBMED ( United States National Library of Medicine) e Google Acadêmico. Baseado na revisão de literatura pré-existentes, desde sua fase técnico-científica até o resultado prático. Foram utilizados trabalhos de 2009 a 2021 com os descritores “ CAD/CAM", “cerâmica", "estética dental"e " reabilitação bucal”.

Portanto, trata-se de um estudo descritivo, qualitativo feito por meio da técnica de observação direta, sendo o pesquisador o instrumento primordial (Pereira et al.,2018).

Os dados do paciente foram coletados através de anamnese realizada na clínica de reabilitação oral da Universidade Estadual do Oeste do Paraná. Seguindo os princípios éticos o paciente consentiu com a divulgação dos dados e exibição de imagens com finalidade acadêmica por meio da assinatura de um Termo de Consentimento Livre e Esclarecido.

\section{Relato de Caso}

Paciente J.B.H, do sexo feminino, 55 anos, leucoderma, compareceu ao programa de Residência em Reabilitação Oral da Universidade Estadual do Oeste do Paraná, queixando-se de sua aparência, principalmente por ter os dentes muito pigmentados.

Inicialmente, foi realizada anamnese e exame clínico, constatou-se presença de múltiplas restaurações na bateria anterior superior e presença de tratamento endodôntico nos elementos 12 e 21. Optou-se por substituir estas restaurações em um primeiro momento, por mostrarem sinais de infiltração e serem consideradas antigas; a paciente não soube relatar em que momento foram realizadas (Figura 1).

Figura 1 - Sorriso inicial evidenciando as deficiências estéticas.

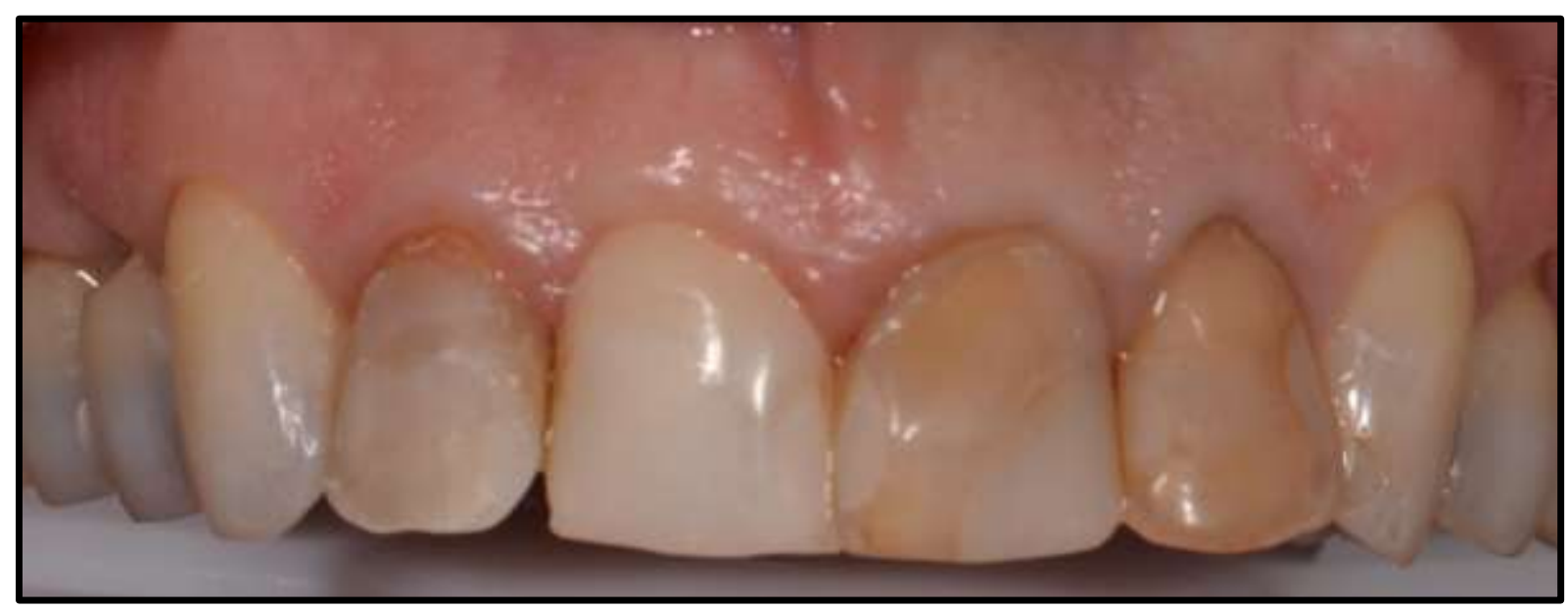

Fonte: Autores. 
O processo reabilitador se iniciou com 2 sessões de clareamento dental assistido com peróxido de hidrogênio à 35\% (HP Maxx - FGM) (Figura 2).

Figura 2 - Clareamento com peróxido de hidrogênio.

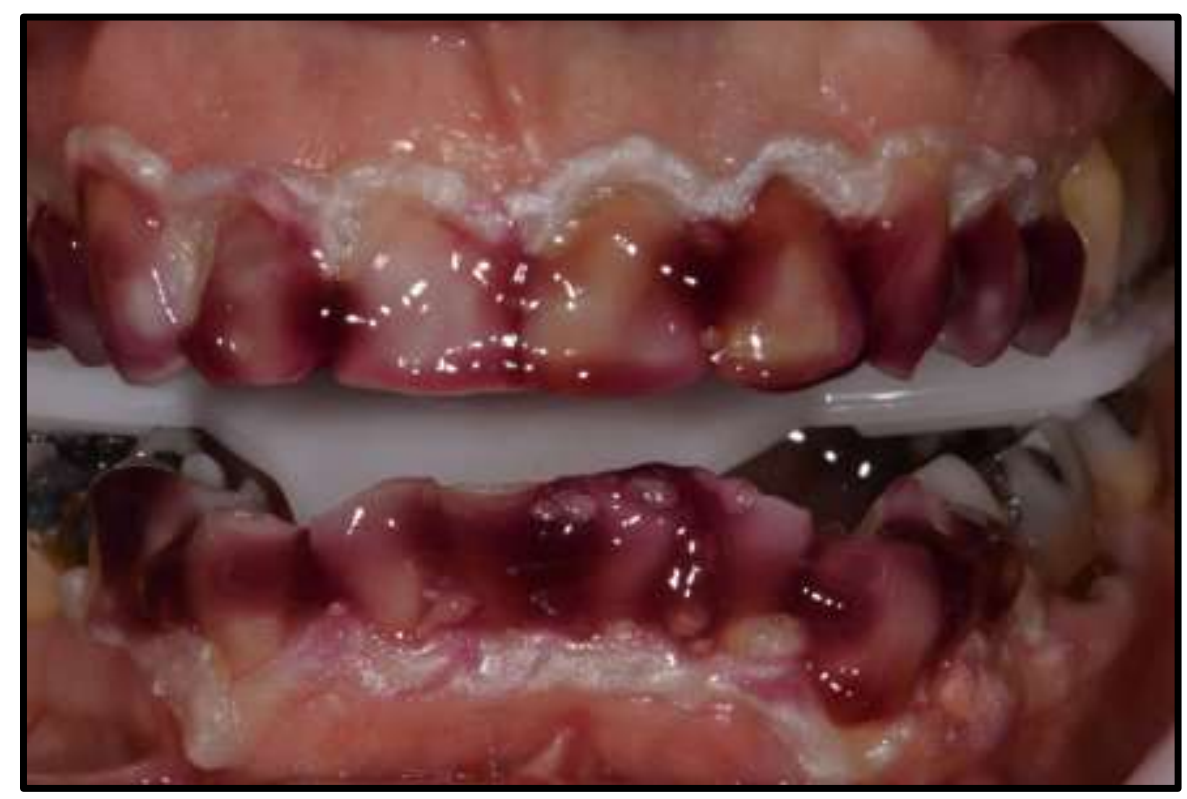

Fonte: Autores.

Após a substituição das restaurações antigas, foi realizado um primeiro escaneamento para armazenar a condição inicial observada e termos como um modelo de estudo digitalizado (Figura 3).

Figura 3 - Modelo de estudo digitalizado.

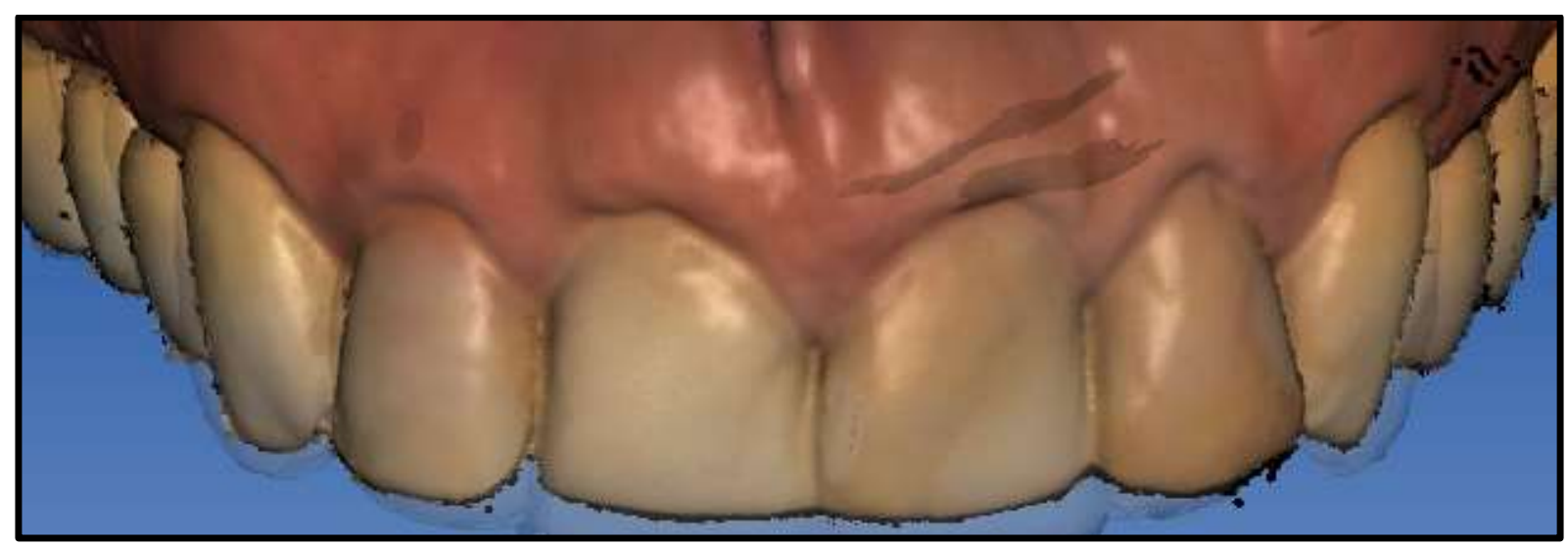

Fonte: Autores.

Depois desse primeiro escaneamento, realizou-se um ensaio restaurador de adição (mock-up) em resina composta que ficasse de agrado para a paciente, então, esse planejamento inicial também foi escaneado em posição intra-bucal para armazenamento da cópia biogenérica na biblioteca do software (CEREC in Lab), com objetivo de apoio e base para a confecção das restaurações finais em cerâmica (Figura 4). 
Figura 4 - Mock-up; A vista frontal; B vista lateral; C vista superior.

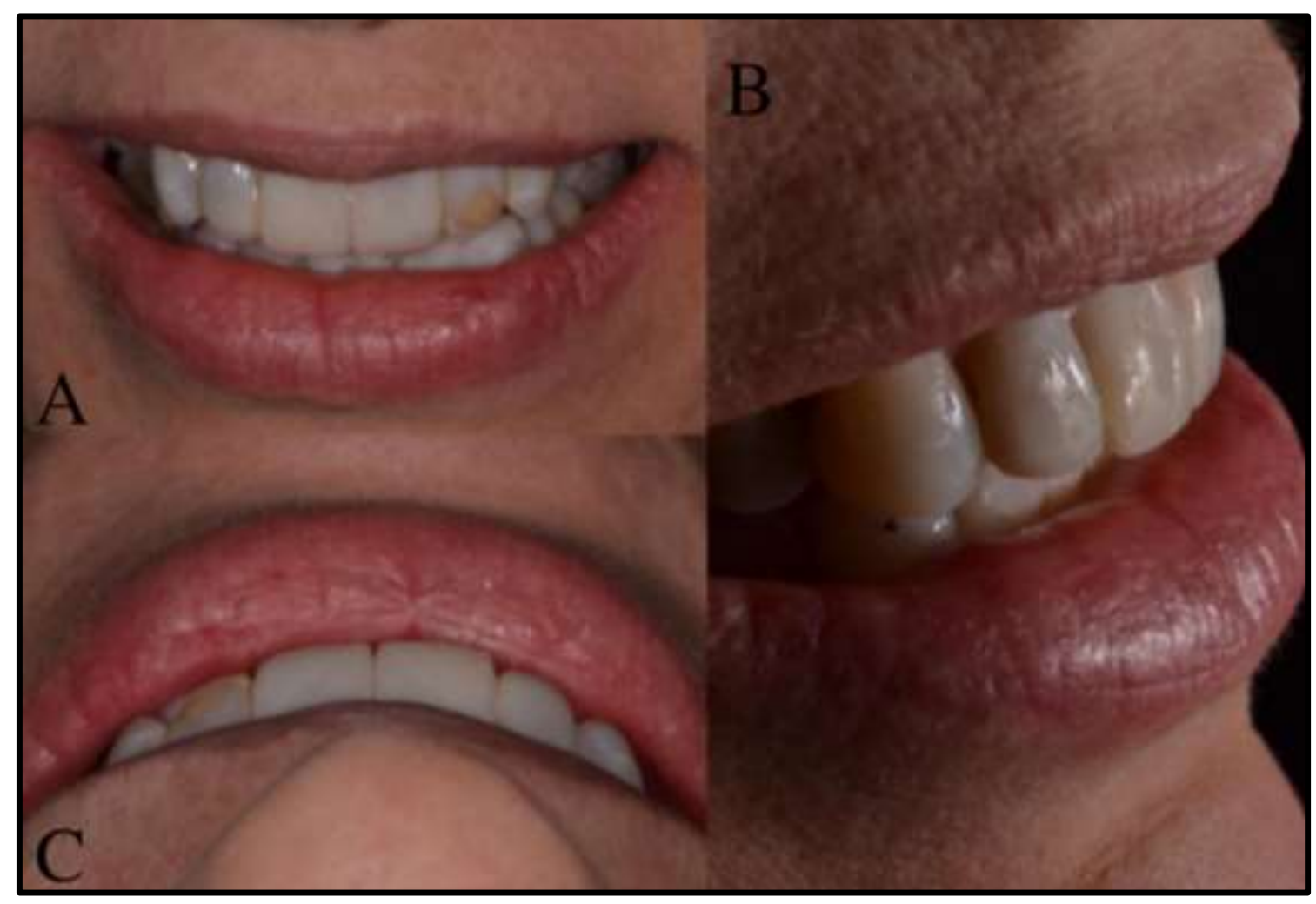

Fonte: Autores.

Depois de aguardar 21 dias para total liberação do oxigênio presente nos radicais livres na hidroxiapatita, realizou-se os preparos dos elementos dentais, sobre o mock-up novamente em um segundo momento, para maior precisão na espessura do desgaste, sempre optando por preservar ao máximo a estrutura dental.

Os dentes foram preparados com o mínimo de desgaste possível, sendo os dentes 12 e 22 preparados para coroa total e portanto, com preparos de maior espessura, uma média de $1,6 \mathrm{~mm}$ de desgaste. Dente 21 preparado para faceta com substrato escurecido, o desgaste teve uma média de $0,9 \mathrm{~mm}$ e nos dentes 13,11 e 23 que não possuíam escurecimento dental, o desgaste ficou na média de $0,6 \mathrm{~mm}$ respeitando a vitalidade dos que ainda possuíam (Figura 5).

Figura 5 - Preparos para facetas digitalizados.

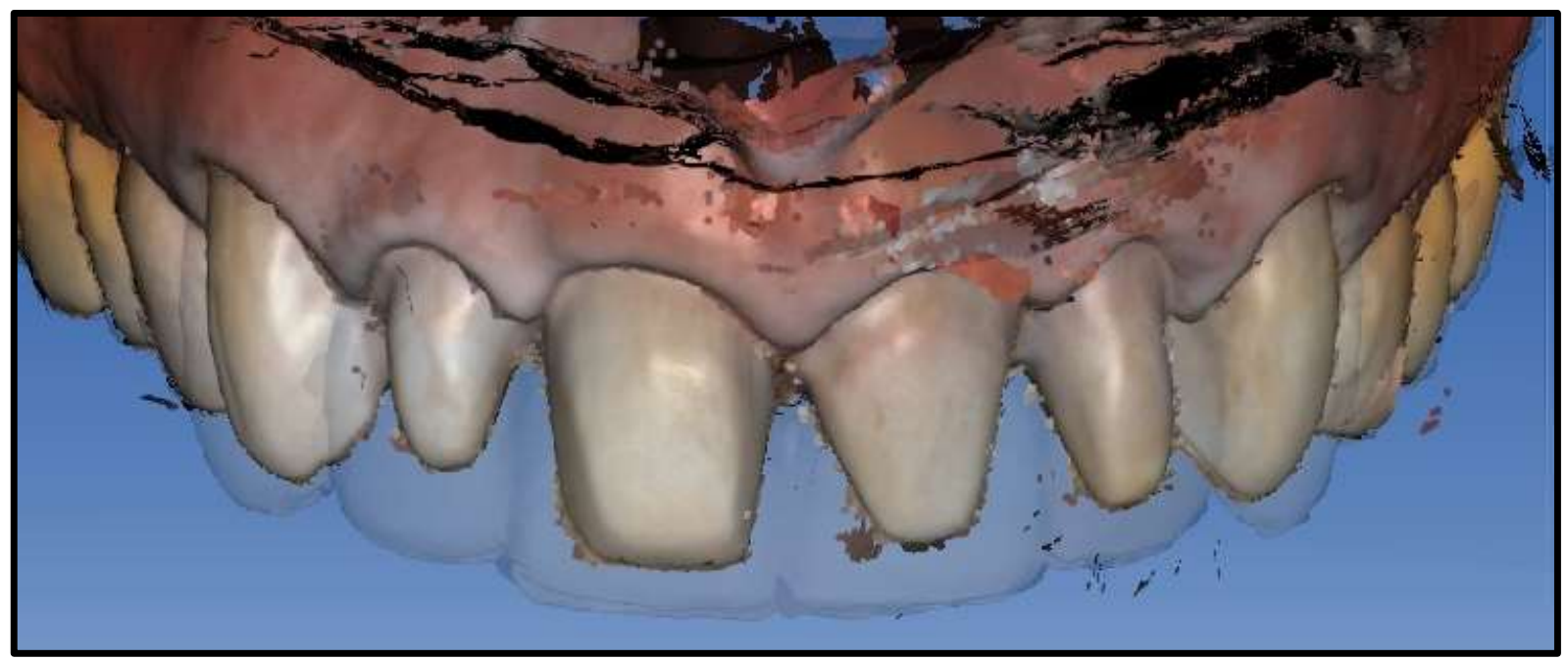

Fonte: Autores. 
Após a realização dos preparos, estes, foram escaneados para iniciar a fase de design das peças, utilizando a opção de cópia biogenérica para então as peças cerâmicas serem confeccionadas (Figura 6).

Figura 6 - Escaneamento.

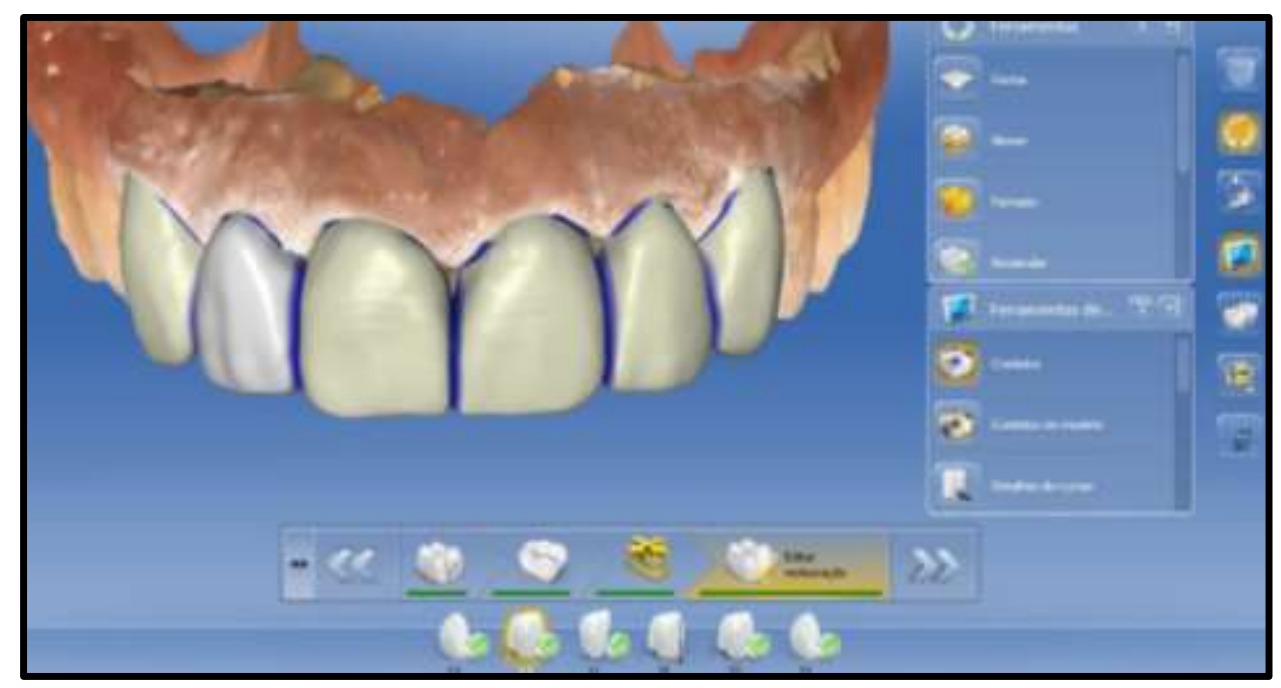

Fonte: Autores.

As etapas convencionais na confecção de uma faceta ou coroa em laboratório, se dão por: recorte do troquel, delimitação da margem, estabelecimento do eixo de inserção, análise da superfície do preparo. Todas essas etapas são imprescindíveis e também são realizadas no software, porém o mesmo ainda complementa com ferramentas que auxiliam ainda mais na confecção dessas restaurações como: análise de parâmetros, análise da infraestrutura, alimentando o programa a fim de permitir que o software crie um design, com pressão nos pontos de contatos adjacentes e antagônicos satisfatórios, análise superfície mínima conforme material utilizado, forma da borda cervical da prótese, espessura do cimento, análise superfície do preparo; que é o diferencial do mesmo.

Outra ferramenta interessante do software é a precisão na posição de oclusão, sendo essa precisão superior ao sistema analógico, onde partimos de modelo de gesso e montagem em articulador.

A escolha de cor do substrato pode ser realizada de forma convencional, visualizando as escalas dos blocos como foi realizado no presente caso, ou até mesmo com auxílio de uma escala própria de dentes escurecidos (Die Natural IPS), que é uma escala criada pela própria ivoclar vivadent; esta, permite que o substrato seja visualizado a fim de escondê-lo total ou parcialmente após a cimentação das restaurações.

Além da escala citada, também é possível prever virtualmente qual a melhor escolha de blocos para ser usado com auxílio de um aplicativo para smartphones chamado de IPS e.max Shade navigation App, quando for utilizado blocos de dissilicato de lítio. Este aplicativo permite que a partir da cor do substrato tomado com a escala Die Natural IPS e da espessura da peça, seja escolhido o melhor bloco com transparência e/ou opacidade para mascarar o substrato.

Optou-se por utilizar uma cerâmica monolítica de média translucidez (MT) da cor A2 do bloco para CAD/CAM IPS E.max CEREC inlab - Ivoclar Vivadent para se obter um equilíbrio da opacidade, já que neste caso em específico existia uma gama de substratos divergentes entre si (Figura 7). 
Figura 7 - Bloco para CAD/CAM.

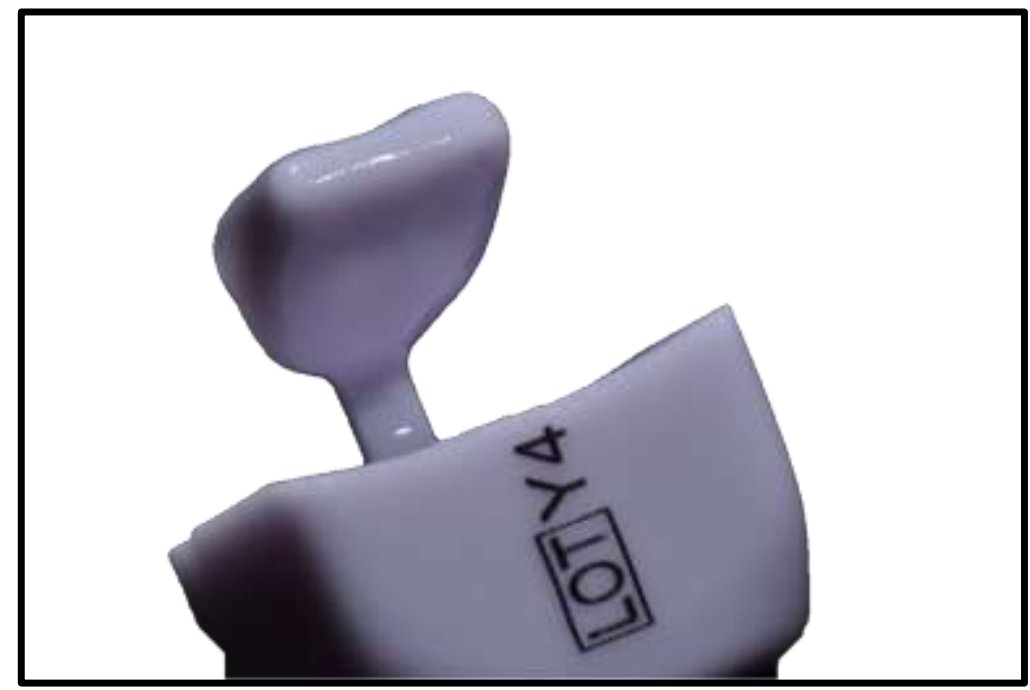

Fonte: Autores.

Após a fresagem da peça, realizou-se a prova dos elementos antes da cristalização dos mesmos, essa prova é de grande importância para melhorar ainda mais o resultado final, podendo ser avaliado, tamanho, forma, contorno além da adaptação marginal. A cerâmica nessa fase é menos resistente, facilitando na hora dos ajustes.

Depois de ter realizado a prova, o dissilicato foi colocado no forno à $850^{\circ}$ para concluir o processo de cristalização que é o processo de tratamento térmico dos cristais de dissilicato, quando estes aumentam de tamanho, número e forma enquanto a matriz vítrea é evaporada, tendo uma alteração microestrutural que resulta em propriedades mecânicas melhoradas (Figura 8).

Figura 8 - Resultado final.

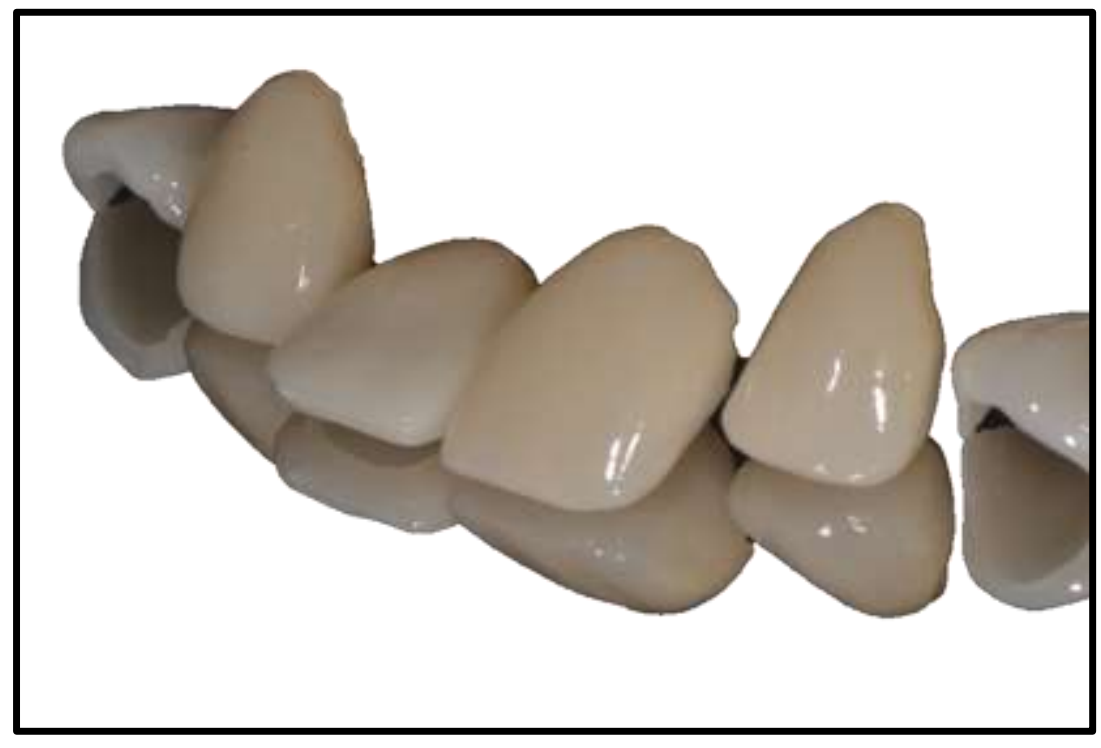

Fonte: Autores.

Após o processo de cristalização, iniciou-se a maquiagem das peças cerâmicas que se deu pelo Pigmento Para Porcelana Essence E01 White Ivocolor - Ivoclar Vivadent,sendo que nos elementos 12 e 22 foi utilizado quatro camadas de pigmento branco, nos elementos 11 e 21 três camadas e nos elementos 13 e 23 duas camadas do mesmo pigmento, sendo esses pigmentos queimados a $350{ }^{\circ} \mathrm{C}$ no forno de mircroglaze (sem vácuo) da fabricante Odontomega ${ }^{\circledR}$, depois da queima dos pigmentos, foi queimado o glaze final na peça para o brilho de superfície (Figura 9). 
Figura 9 - Cerâmicas monolíticas.

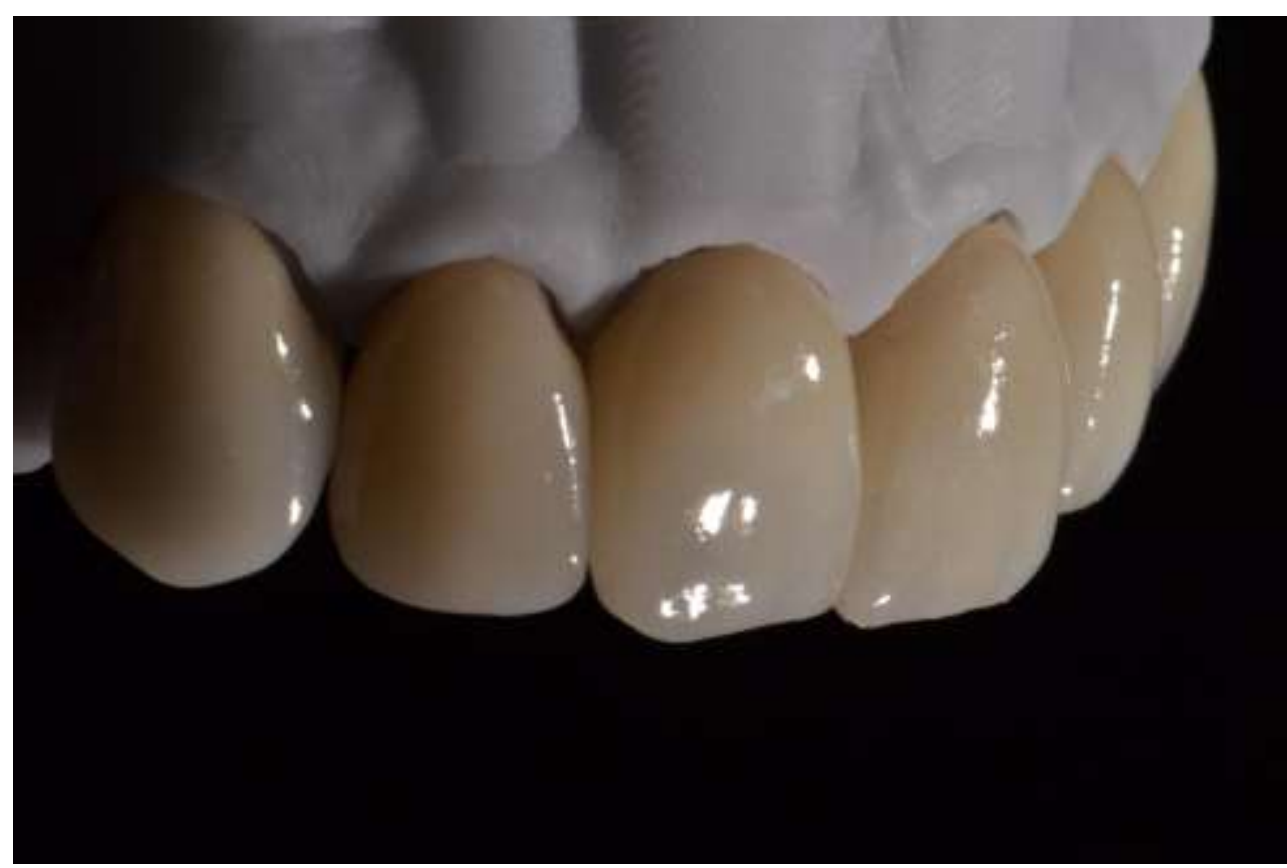

Fonte: Autores.

É importante relatar que não foi utilizado recurso de maquiagem para finalizar a peça, apenas branco para aumentar o valor e o glaze para fluorescência - Empress Multi.

Após, realizou-se o processo de cimentação, optando-se por isolamento relativo, e pela utilização de um cimento dual convencional, RelyX Ultimate 3M SPEE. As peças foram preparadas com jateamento de óxido de alumínio, ácido fluorídrico 10\% a 20 segundos, silanização com o agente Monobond - Ivoclar Vivadent e uma camada fina de adesivo Single Bond Universal - 3M. Já o dente foi preparado com profilaxia com pedra pomes, ácido fosfórico 37\% em esmalte e sistema adesivo Single Bond Universal - 3M em esmalte e dentina, sendo que na última foi aplicado vigorosamente, como sugere o fabricante; após todo esse preparo, foi manipulado e aplicado o cimento resinoso. As peças então foram levadas às posições, removidos os excessos e fotopolimerizada por 40 segundos cada face de cada dente (Figura 10).

Figura 10 - Resultado final imediato.

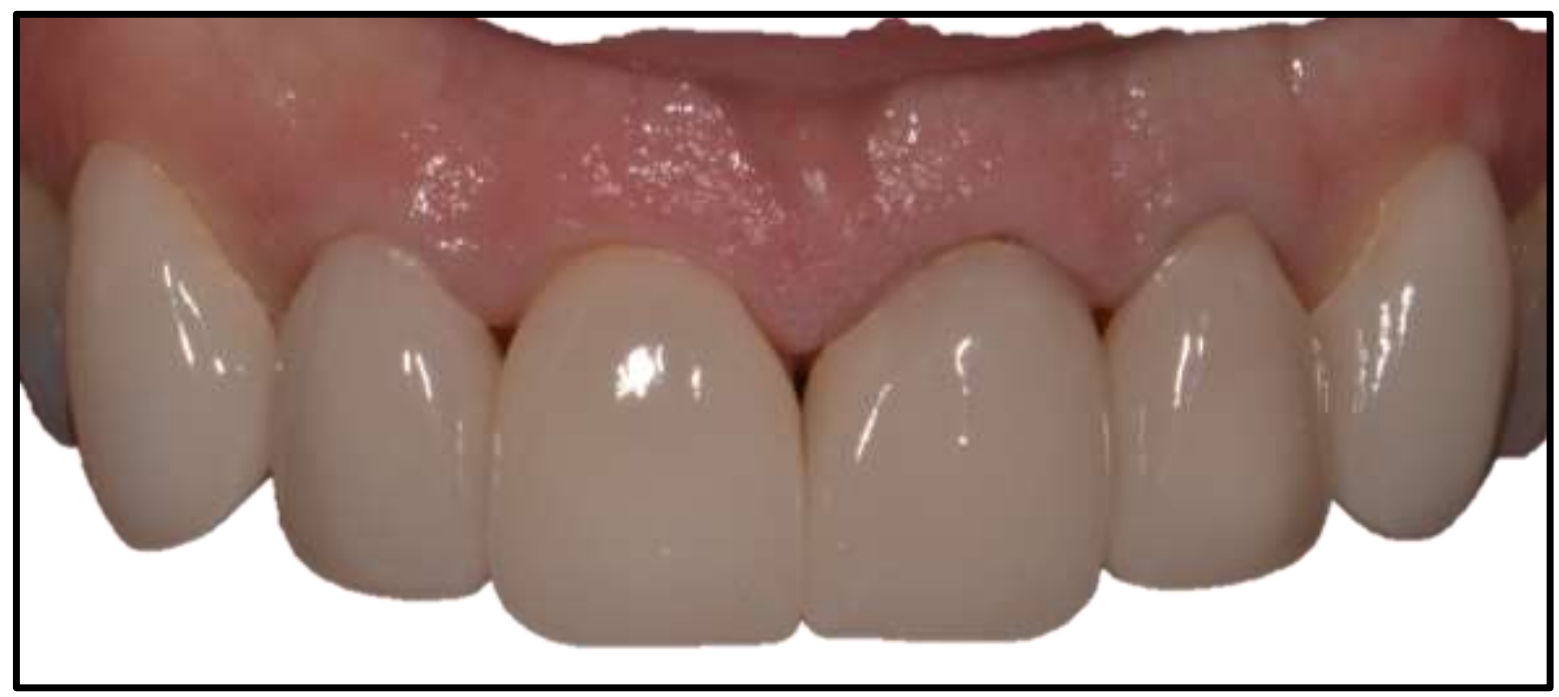

Fonte: Autores. 


\section{Discussão}

O processo de seleção de cor é um fator fundamental no sucesso de um tratamento restaurador estético. Atualmente, através do avanço tecnológico alcançado em termos de aferição de cor, é possível utilizar instrumentos (colorímetros, e espectrofotômetros ou espectroradiômetros) com o objetivo de fazer uma seleção de cor com maior precisão (Maroli, 2019).

As facetas laminadas e coroas totais cerâmicas estão indicadas para os casos onde exista a necessidade de alteração de cor ou de forma das faces vestibular e incisal dos dentes (Chang, et al, 2009). Quando utilizadas sobre substratos escurecidos, as facetas e coroas necessitam apresentar equilíbrio entre sua capacidade de mascarar o substrato e o resultado estético final. Um aumento da espessura da restauração e o emprego de cerâmicas de maior opacidade colaboram no mascaramento. Porém, o aumento de espessura incorre, obrigatoriamente, em preparos dentários mais invasivos, o que reduz a quantidade de esmalte disponível para os procedimentos adesivos, diminuindo, assim, a expectativa de sucesso clínico da faceta de cerâmica (Chang, et al, 2009). Cerâmicas demasiadamente opacas são incapazes de simular o comportamento óptico do esmalte dentário que é caracterizado pela sua translucidez, emprestando às restaurações um aspecto artificial, esteticamente desagradável (Hilgert, 2009).

Tem sido relatado que quanto maior a opacidade maior a saturação da cerâmica, menor a transmissão da luz, o que resulta em propriedades físico-químicas inferiores dos agentes de cimentação resinosos (Archegas et al., 2012; Calgaro et al., 2013; Passos et al., 2013; Leal et al., 2016); sendo observado que a cerâmica de dissilicato de lítio, de menor translucidez, ocasionou menor grau de conversão dos agentes de cimentação, quando comparado à cerâmica de maior translucidez. A menor translucidez promove dissipação da luz, e desta forma menor intensidade de luz atinge o agente de cimentação (Archegas et al., 2012; Passos et al., 2013). Assim, nos casos de reabilitações de dentes mais escurecidos em que se faz necessário o uso de cerâmica de maior opacidade tem sido recomendado se prolongar o tempo de fotoativação (Archegas et al., 2012).

No estudo realizado por Guarnieri et al. 2014 constatou-se que com cerâmicas de média opacidade conseguiu-se alteração de cor significante (para mais clara) somente para as espessuras de $0,7 \mathrm{~mm}$, enquanto que com cerâmicas de baixa translucidez (mais opacas), a alteração foi mais significante (para mais clara) tanto em espessuras de $0,3 \mathrm{~mm}$ como de $0,7 \mathrm{~mm}$, sugerindo, portanto, que laminados muito finos $(0,3 \mathrm{~mm})$ necessitam de opacidade alta para mascarar cores de maneira significante.

A grande dificuldade de quem almeja ingressar nessa tecnologia é provavelmente entender como a incorporação destas se encaixa em cada modelo de negócio. Como utilizar esta nova ferramenta diante das necessidades de cada caso.

O desajuste marginal médio em restaurações usando sistema CAD/CAM fica entre 23,08 mm a 36,11 mm, podendo ser considerado clinicamente satisfatório (Dartora et al., 2014).

Para realizar trabalhos de excelência, algumas etapas tradicionais do processo precisam ser mantidas, como o enceramento de diagnóstico (Adolfi, 2016).

A atual complexidade dos materiais dificulta a decisão do dentista e do técnico em prótese dentária na escolha dos materiais restauradores, assim como, as diferenças entre o sensor do scanner e as limitações do olho humano para perceber sutilezas de coloração nas próteses (Adolfi, 2016).

\section{Conclusão}

A partir do relato de caso apresentado, temos que o sistema CAD/CAM é de positiva evolução na odontologia por promover restaurações de forma ágil e com bons padrões de qualidade. $O$ desafio que existe entre o mascaramento do substrato é eminente, mas este pode ser contornado indicando uma cerâmica com bom fator de opacidade e/ou translucidez, quanto mais opaco, mais recobre o substrato menor a naturalidade da peça capaz de simular o comportamento óptico do esmalte dentário. etapas clínicas alcançadas com sucesso, ótimas adaptações das peças, mimetização das estruturas dentais, resultando em 
restaurações cerâmicas de excelência, bem indicadas, confiáveis e esteticamente agradáveis, que foi o principal motivo pelo qual a paciente procurou atendimento.

Algumas limitações a respeito do sistema CAD/CAM devem ser consideradas pelo profissionais antes de sua utilização; apesar de ser uma tecnologia muito utilizada pela engenharia, arquitetura e manufatura ainda faltam estudos que comparem a fidelidade do resultado final com os processos laboratoriais convencionais e os utilizados pelo sistema CAD/CAM, assim como acompanhamentos clínico e radiográfico a longo prazo do uso desse sistema em reabilitações orais extensas, principalmente em regiões posteriores.

\section{Referências}

Alves, V. (2017). Vantagens x desvantagens do sistema CAD/CAM. Brazilian Journal of Surgery and clinical Research, 18(1), 106-109.

Chang, J., Da Silva, J. D., Sakai, M., Kristiansen, J., \& Ishikawa-Nagai, S. (2009). The optical effect of composite luting cement on all ceramic crowns. Journal of dentistry, 37(12), 937-943.

Correia, A. R. M., Fernandes, J. C. A. S., Cardoso, J. A. P., \& Silva, C. F. C. L. (2013). CAD-CAM: a informática a serviço da prótese fixa. Revista de Odontologia da UNESP, 35(2), 183-189.

Cruz, E. M. (2018). Sistemas CAD/CAM na Odontologia.

Dartora, G., Dartora, N., Radaelli, M. B., Alessandretti, R., Spazzin, A. O., \& Bacchi, A. (2014). Precisão dos sistemas CAD/CAM em restaurações unitárias: revisão de literatura. Prosth. Lab. Sci, 3(10), 133-139.

Hilgert, L. A. (2009). Influência da coloração do substrato, espessura e translucidez da cerâmica na cor de facetas laminadas produzidas com o sistema CEREC InLab.

Maroli, A., Federizzi, L., Spazzin, A. O., \& Radaelli, M. T. B. (2019). Comparação estética entre sistemas cerâmicos sobre substrato escurecido. Journal of Oral Investigations, 8(1), 69-85.

Martins, L. D. M. (2011). Confiabilidade de coroas de dissilicato de lítio com diferentes espessuras sob fadiga (Doctoral dissertation, Universidade de São Paulo).

Moura, R. B. B., \& Santos, T. C. (2015). Sistemas cerâmicos metal free: tecnologia CAD/CAM-revisão de literatura. Revista Interdisciplinar, 8(1), $220-226$.

Pedroche, L. O., Bernardes, S. R., Leao, M. P., Kintopp, C. C. D. A., Correr, G. M., Ornaghi, B. P., \& Gonzaga, C. C. (2016). Marginal and internal fit of zirconia copings obtained using different digital scanning methods. Brazilian oral research, 30(1).

Pereira, A. S., Shitsuka, D. M., Parreira, F. J., \& Shitsuka, R. (2018). Metodologia da Pesquisa Científica-Licenciatura em Computação. UFSM. 